\title{
Arquitectura de referencia para aplicaciones con tecnología NFC basadas en Android
}

\author{
Alejandra Anaya, Ignacio López, Celia Romero, \\ Beatriz Olivares, Hilarión Muñoz \\ Instituto Tecnológico de Orizaba, Veracruz, México \\ alejandra_anaya@acm.org \{ilopez, cromero, bolivares\}@ito-depi.edu.mx, \\ hmunozc198@msn.com
}

\begin{abstract}
Resumen. NFC (Near Field Communication, Comunicación de campo cercano) es el resultado de la integración de distintas tecnologías, tales como RFID, tarjetas inteligentes y de comunicación inalámbrica, lo cual le brinda una ventaja evidente al ser compatible con las mismas. Actualmente se ha vislumbrado la potencialidad de esta tecnología y el desarrollo de aplicaciones NFC está incrementando considerablemente, sin embargo, no se documentan arquitecturas de referencia en el contexto de NFC que permitan el desarrollo de aplicaciones en un tiempo más corto y apoyen en la estandarización y evolución de este tipo de aplicaciones. Por tal motivo en el presente trabajo se propone una arquitectura de referencia para el desarrollo de aplicaciones NFC basadas en el Sistema Operativo Android y se presenta la implementación de un caso de estudio aplicando la arquitectura propuesta.
\end{abstract}

Keywords: NFC, arquitectura de referencia, aplicaciones NFC, android.

\section{Introducción}

NFC es una tecnología de comunicación inalámbrica relativamente nueva; surgió en el año 2002 y fue creada por la compañía Sony Philips, sin embargo, fue hasta principios del año 2009 cuando alcanzó un uso más amplio debido a su integración en teléfonos celulares, abarcando aplicaciones en distintas áreas, tales como medicina, control de accesos, publicidad, educación, identificación, control de activos, pagos móviles, por mencionar algunos [1]. Sus principales características son: 1) comunicación de corto alcance (máximo $10 \mathrm{~cm}$.) lo que brinda a NFC una seguridad implícita en el intercambio de información crítica; 2 ) alta frecuencia en la velocidad de transmisión de datos (424 Kbits por segundo) ya que está diseñada para transmitir pequeñas cantidades de información rápidamente; 3) facilidad de uso al brindar al usuario una manera intuitiva de utilizarla; 4) compatibilidad con otras tecnologías existentes debido a que su principal objetivo es complementarlas [2].

A partir de la integración de la tecnología NFC en los teléfonos celulares se comenzaron a realizar proyectos de prueba para realizar pagos con móviles utilizando esta tecnología, y se incrementó significativamente el desarrollo de aplicaciones en 38 países con resultados exitosos [3], asimismo existen documentadas algunas 
arquitecturas de aplicaciones NFC, sin embargo, dichas arquitecturas son propias al sistema a desarrollar, y no se encuentran documentadas arquitecturas de referencia para el desarrollo de este tipo de aplicaciones. Por otra parte las arquitecturas de referencia proveen una base para el desarrollo de aplicaciones dentro de un dominio específico al definir un conjunto de componentes de construcción [4]; es por este motivo, que el objetivo del presente artículo es dar a conocer una arquitectura que defina los elementos arquitectónicos abstractos de un sistema NFC, con la finalidad de obtener arquitecturas de software dentro del dominio de la tecnología NFC y lograr la implementación de aplicaciones en un tiempo relativamente más corto. Por otro lado contar con una arquitectura de referencia es de suma importancia para homogeneizar el desarrollo de este tipo de aplicaciones y así lograr una interacción transparente entre distintas tecnologías.

El presente artículo se encuentra estructurado de la siguiente manera: en la sección 2 se mencionan brevemente algunos trabajos relacionados, posteriormente en la sección 3 se define la arquitectura propuesta y se describen los componentes que la conforman; en la sección 4 se presenta la implementación de un caso de estudio como prueba de concepto utilizando la arquitectura propuesta, el cual consiste en una aplicación Android con tecnología NFC para gestionar el préstamo de libros de la biblioteca de posgrado del Instituto Tecnológico de Orizaba, finalmente en la sección 5 se mencionan las conclusiones y trabajos a futuro.

\section{Trabajos relacionados}

A lo largo de los últimos años se han realizado diferentes proyectos dentro del contexto de la tecnología NFC, además, estos proyectos proveen información acerca de sus conceptos principales, Paus Anikka et al. [5] definen a NFC como una tecnología de comunicación inalámbrica de corto alcance entre dispositivos que la soporten, utilizando la inducción de campo magnético para establecer dicha comunicación. En [6] se indica que el funcionamiento de NFC es bastante simple ya que únicamente intervienen 2 dispositivos; al que inicia la comunicación se le llama "iniciador" y el que responde se le denomina "objetivo", la comunicación se inicia cuando los dispositivos se acercan el uno al otro a una distancia muy corta, máximo de $10 \mathrm{~cm}$. Por otro lado Paus Anikka et al. [5] afirman que la característica de corto alcance de NFC no es una desventaja, porque brinda una mayor seguridad en la transferencia de datos y Riyazuddin et al. [7] enfatizan el uso de SSL (Secure Sockets Layer, Capa de Conexión Segura) para codificar y transmitir dichos datos de manera segura. Con base en un análisis comparativo realizado en [2] entre NFC y otras tecnologías de comunicación inalámbrica tales como Bluetooth e IrDa (Infrared Data Association, Asociación de Datos Infrarrojos) se identificaron los siguientes beneficios a favor de NFC: Fácil configuración, compatible con infraestructuras RFID, seguro, de bajo costo, entre otros; cabe mencionar que la facilidad en el uso y configuración de NFC es una característica muy importante porque brinda a los usuarios una manera transparente e intuitiva de interactuar con dicha tecnología y otras ya existentes.

Con respecto a aplicaciones de NFC en [8] se presenta un sistema utilizando etiquetas NFC y el teléfono celular Nokia 6212 para rastrear el uso de cloro en el agua potable de los hogares en Haití. Las etiquetas NFC se adhirieron a los contenedores de 
agua en los hogares para que los trabajadores (que realizaban visitas en las casas periódicamente, midiendo manualmente la cantidad de cloro en el agua) únicamente acercaran el celular a la etiqueta NFC y mediante una aplicación en el celular actualizaran la cantidad de cloro y una serie de medidas necesarias que anteriormente se registraban en hojas de papel. En [9] se presenta un caso de estudio utilizando un teléfono celular con hardware TEE (Trusted Execution Environment, Entorno de Ejecución Confiable) para la identificación de boletos de transporte haciendo énfasis en aspectos de seguridad y desempeño. Se describe la arquitectura utilizada y se realiza un análisis de las propiedades de seguridad proporcionadas por la arquitectura propuesta. Para lograr la comunicación entre el lector de boletos y el teléfono se utilizó LLCP (Logical Link Control Protocol, Protocolo de Control de Enlace Lógico) que permite el intercambio de datos entre dos dispositivos. G. Madlmayr et al. [10] afirman que a pesar de que NFC brinda al usuario final una interacción simple, la infraestructura detrás de este escenario es bastante compleja y se requiere de distintos mecanismos para asegurar la interoperabilidad de las aplicaciones, por lo tanto proponen una arquitectura para gestionar aplicaciones de tarjetas inteligentes de manera segura en los dispositivos NFC.

En [11] se define a una arquitectura de referencia como un marco común, el cual se personaliza para obtener arquitecturas dentro de un dominio de aplicación específico; además se presenta una arquitectura de referencia para el procesamiento de eventos.

En [12] se discute la importancia que poseen los componentes y la composición en la definición de arquitecturas de referencia y se menciona la manera en la que el diseño basado en componentes contribuye significativamente en la definición y uso de este tipo de arquitecturas en la práctica, además se enfatiza que los puntos de variación de una arquitectura son las relaciones entre las características padre-hijo dentro del modelo.

Como se observa en los trabajos anteriormente examinados, se identifican algunas cuestiones como: a) En [9] y [10] se presentan arquitecturas de las aplicaciones NFC, sin embargo estas son propias del sistema que se presentó, es decir, no son arquitecturas de referencia. b) En [11] y [12] se proponen arquitecturas de referencia, no obstante éstas son de dominios de aplicación diferentes a la tecnología NFC, por lo tanto el presente trabajo busca solventar estas deficiencias al definir una arquitectura de referencia para aplicaciones NFC y reforzarla implementando un caso de estudio utilizando la arquitectura propuesta.

\section{Arquitectura}

En esta sección se presenta la definición de la arquitectura, que se basa en los patrones arquitectónicos Modelo-Vista-Controlador y SOA (Service-Oriented Architecture, Arquitectura Orientada a Servicios) [13].

\subsection{Planteamiento de la arquitectura}

La figura 1 muestra las capas de la arquitectura propuesta, y se describen las responsabilidades de cada una de las mismas. La funcionalidad de la tecnología NFC se ubica en la capa cliente. 


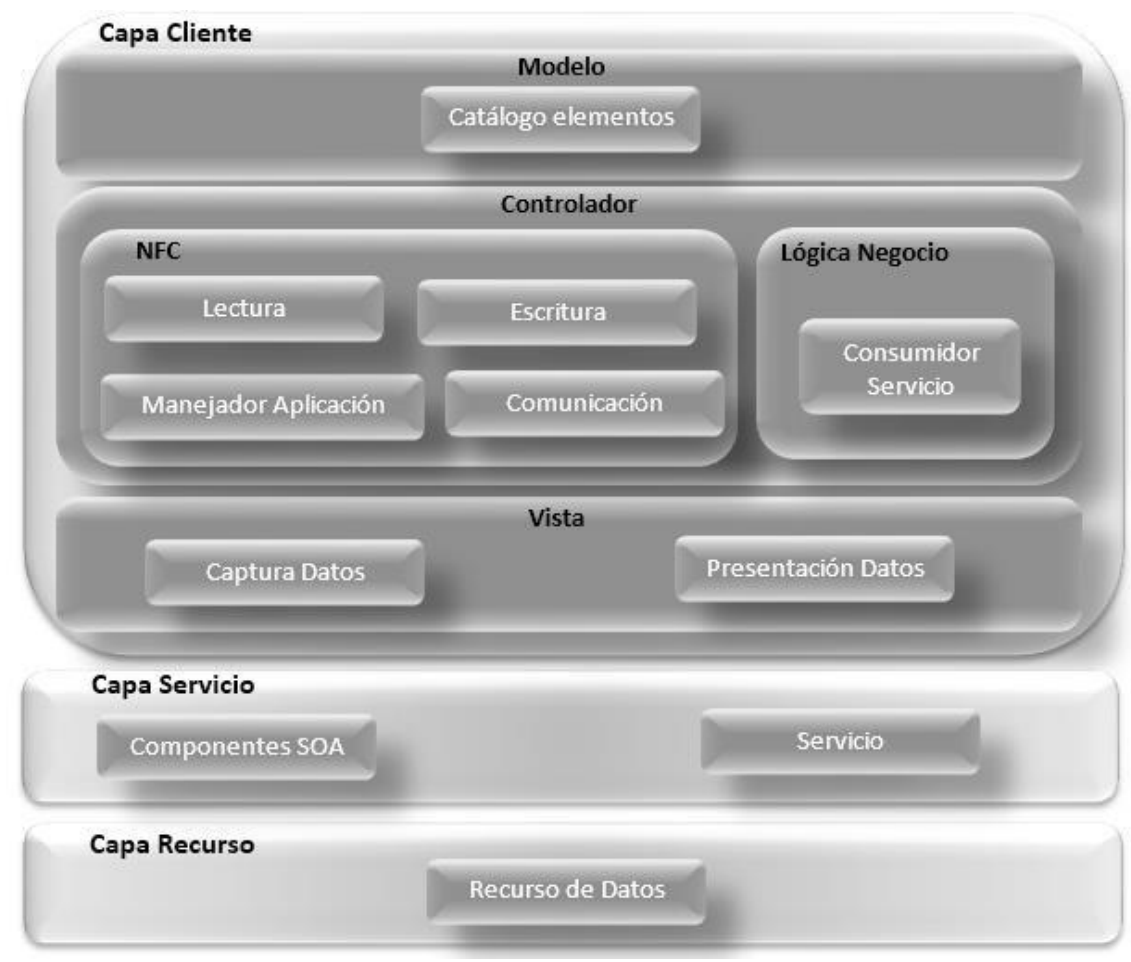

Fig. 1. Arquitectura de referencia para aplicaciones NFC.

La capa de modelo del cliente se encuentra conformada de la siguiente manera:

Catálogo de elementos. Se encarga de almacenar en el dispositivo los datos necesarios para el correcto funcionamiento de la aplicación.

La capa de lógica del cliente se encuentra conformada por los siguientes componentes:

Lectura. Componente ubicado dentro del módulo NFC, el cual se encarga de gestionar la lectura de datos de las etiquetas NFC a la aplicación, genera el campo de radiofrecuencia necesario para extraer los datos de las etiquetas y se ocupa de brindar un formato adecuado a los mismos, asimismo interactúa con el componente de comunicación para establecer el enlace del dispositivo con la etiqueta.

Escritura. Componente ubicado dentro del módulo NFC, el cual se encarga de la preparación y manipulación de datos que se requieren escribir en la etiqueta, ya que es necesario que cumplan una serie de requisitos previos, entre los cuales están brindarles el formato adecuado además de especificar el tipo de registro que se va a almacenar, es decir si es una url, texto plano, número telefónico, ubicación, entre otros.

Comunicación. Componente ubicado en el módulo NFC, el cual es el encargado de realizar el enlace entre el dispositivo y la etiqueta, además se encarga de gestionar el adaptador NFC del dispositivo.

Manejador de aplicación. Componente ubicado dentro del módulo NFC, el cual es el encargado de gestionar la ejecución de la aplicación correspondiente cuando el 
dispositivo entra en contacto con una etiqueta NFC y así evitarle la tarea al usuario de buscar y ejecutar la aplicación.

Consumidor de servicio. Componente ubicado dentro del módulo de lógica del negocio, el cual lleva a cabo la invocación del servicio web.

La capa de presentación del cliente cuenta con 2 componentes:

Captura de datos. Se encarga de mostrar las vistas necesarias para la solicitud de datos al usuario.

Presentación de datos. Es el encargado de proporcionar las vistas necesarias para el despliegue de información al usuario.

La capa de servicio está formada por los siguientes componentes:

Componentes SOA: Componente de la Capa de servicios, el cual es el encargado de lograr la interoperabilidad de aplicaciones al brindar la infraestructura SOA necesaria.

Servicio. Componente de la capa de servicios, encargado de atender las peticiones de los consumidores.

La capa de recursos está formada por:

Recurso de datos. Componente de la capa de recursos, encargado de almacenar y proporcionar datos al componente de servicio.

\section{Caso de estudio: BibliotecaNFC}

El caso de estudio que se utilizó para implementar la arquitectura planteada consistió en una aplicación Android para la gestión de auto-préstamo de libros de la biblioteca de posgrado del Instituto Tecnológico de Orizaba. Los actores que intervienen en el sistema son: El alumno y el encargado de la biblioteca, como se muestra en la figura 2.

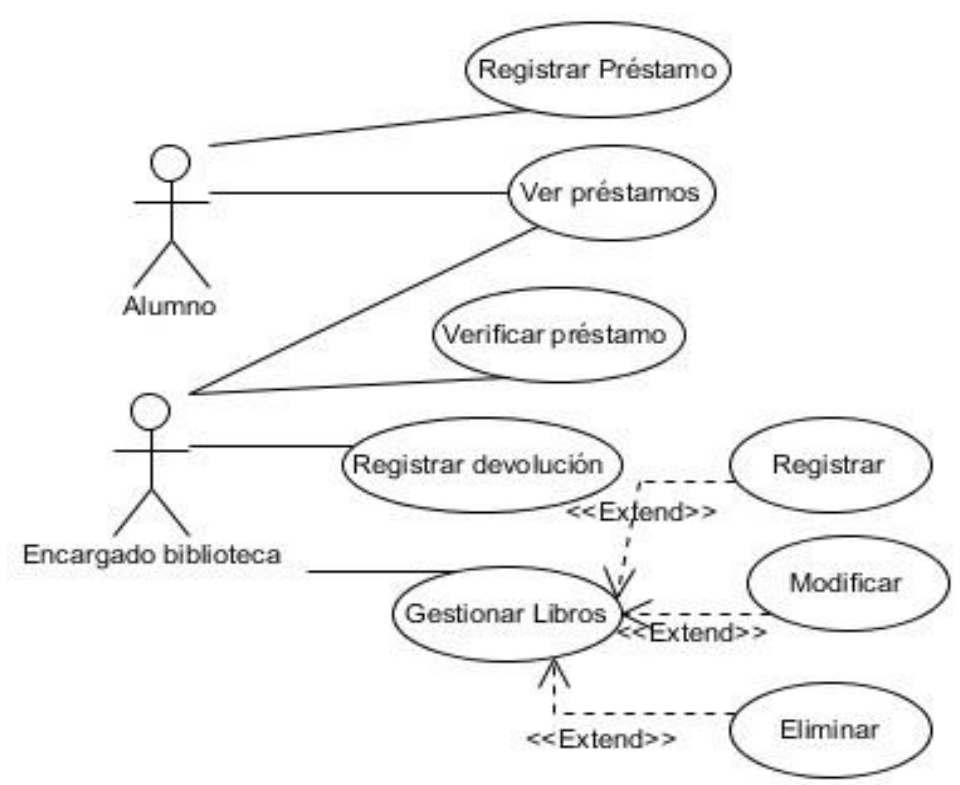

Fig. 2. Diagrama de casos de uso del sistema BibliotecaNFC. 
Ambos utilizan un teléfono celular equipado con tecnología NFC para interactuar con los libros, a los cuales se les adhirió una etiqueta NFC, que contiene información relevante de los mismos. El escenario es el siguiente: 1) El alumno ingresa a la biblioteca y realiza el préstamo de un libro por medio de la aplicación instalada en su teléfono. 2) El encargado de la biblioteca verifica que el alumno efectivamente haya registrado el préstamo del libro y le permite salir de la biblioteca con el respectivo libro. 3) Cuando el alumno regresa el libro a la biblioteca el encargado de la biblioteca registra la devolución del libro. Todas estas funciones se realizan de manera muy sencilla gracias a la tecnología NFC ya que cuando el teléfono celular entra en contacto con la etiqueta del libro la aplicación se ejecuta automáticamente y el usuario simplemente necesita pulsar un botón para realizar cada una de las operaciones correspondientes.

\subsection{Aplicación de la arquitectura}

La distribución de las clases de la aplicación biblioteca NFC empleando la arquitectura propuesta se muestra en la figura 3 , asimismo se observan las relaciones que existe entre clases y el componente de la arquitectura al que pertenecen dichas clases. Como resultado del caso de estudio se obtuvo que la aplicación es fácil e intuitiva, ya que se realizaron pruebas con el usuario final (encargado de la biblioteca) sin ningún tipo de capacitación previa y no tuvo problemas al utilizarla, además se redujo el tiempo en el desarrollo del caso de estudio al poseer una arquitectura definida previamente.

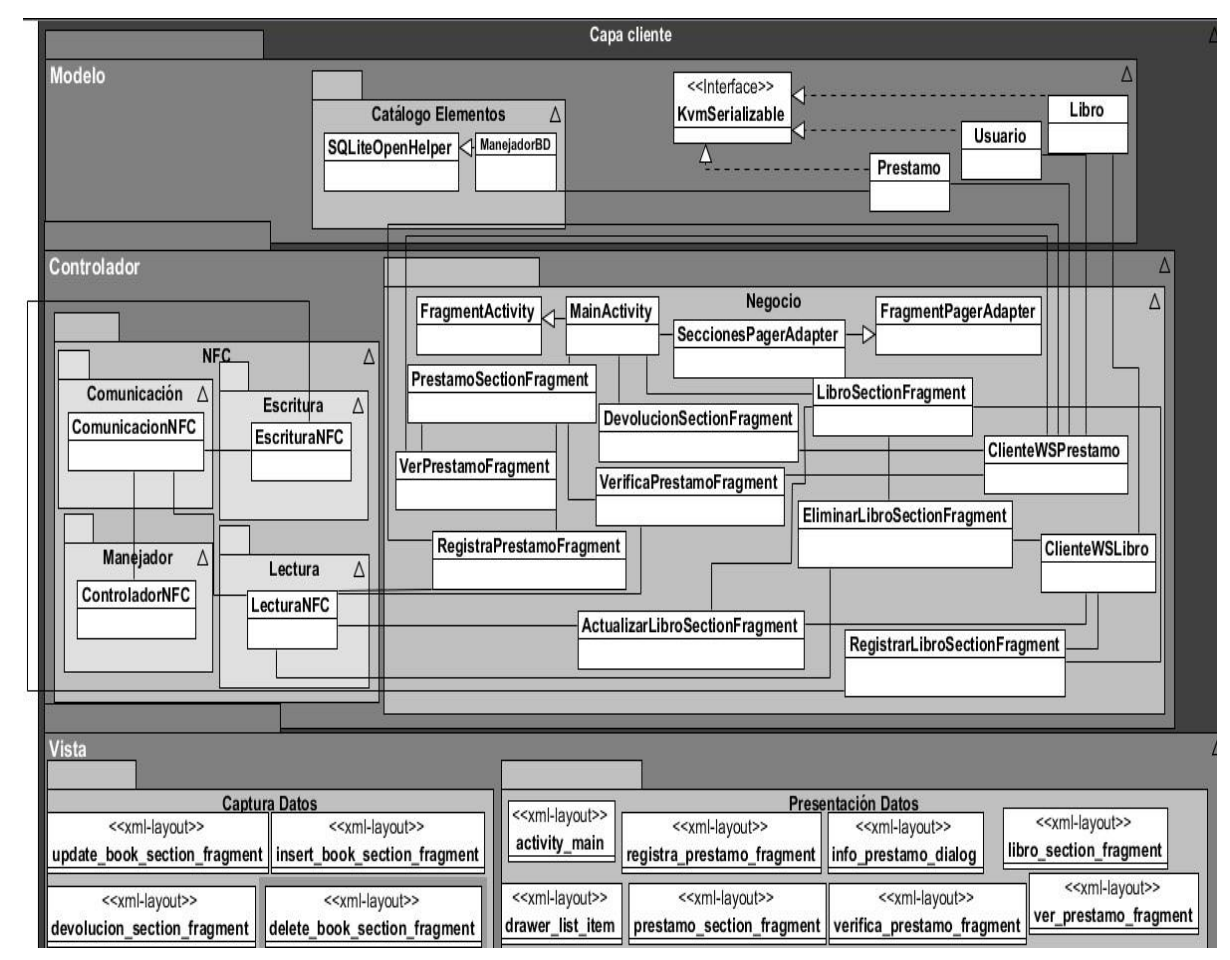

Fig. 3. Capa Cliente arquitectura implementada. 


\section{Conclusiones y trabajos a futuro}

Se presentó la arquitectura de referencia para aplicaciones con tecnología NFC y se implementó un caso de estudio con el objetivo de probar la arquitectura, al ser una tecnología nueva es necesario invertir una cantidad significativa de tiempo en obtener los conocimientos fundamentales para desarrollar aplicaciones en el campo de NFC, por lo tanto contar con una arquitectura de referencia es de suma importancia para agilizar el proceso de desarrollo, por consiguiente la arquitectura propuesta en el presente artículo busca solventar dicha necesidad. Asimismo se realizaron pruebas de usabilidad al responsable de la biblioteca y se obtuvieron resultados exitosos, ya que el usuario no tuvo problemas al utilizar la aplicación.

Como trabajos a futuro se tiene contemplado realizar un segundo caso de estudio para reforzar la arquitectura presentada.

Agradecimientos. Los autores de este artículo agradecemos al CONACyT y a la DGEST por el apoyo y confianza depositados para la investigación y desarrollo del presente.

\section{Referencias}

1. Ok, K., Aydin, M.N., Coskun, V., Ozdenizci, B.: Exploring underlying values of NFC applications. In Proc. of International Conf. on Management (2010)

2. Ok, K., Coskun V., Ozdenizci, B.: Near Field Communication From Theory to Practice. In Proc. of International Conf. on Management Technology and Applications (ICMTA), Singapur, Singapur, pp. 10-12 (2010)

3. Ahson, S.A., Ilyas, M.: Near Field Communications Handbook. Broken Sound Parkway NW, CRC Press, pp.68-75 (2012)

4. Cavalcante, E. Alves, M.P., Batista, T., Delicato, F.C., Pires, P.F.: An Analysis of Reference Architectures for the Internet of Things. pp. 13-16 (2015)

5. Paus, A.: Near field communication in cell phones. In Chair for Communication Security, Vol. 24, p. 8 (2007)

6. E.S., J. Kaartinen, J.P., A. Ylisaukko-oja, I.K.: Application of near field communication for health monitoring in daily life. In Engineering in Medicine and Biology Society, EMBS'06, 28th Annual International Conference of the IEEE, pp. 3246-3249 (2006)

7. Riyazuddin, M.: NFC, a review of the technology, applications and security. (2009)

8. J. Kaye, D. H., E. Seto, B. E., Ritter, M.: Using NFC phones to track water purification in Haiti. In CHI' 12 Extended Abstracts on Human Factors in Computing Systems, pp. 677-690 (2012)

9. S.T., J.-E. Ekberg, N.A.: Identity verification schemes for public transport ticketing with NFC phones. In Proceedings of the sixth ACM workshop on Scalable trusted computing, pp. 37-48 (2011)

10. Madlmayr, G.: A mobile trusted computing architecture for a near field communication ecosystem. In Proceedings of the 10th international conference on information integration and web-based applications \& services, pp. 563-566 (2008)

11. K.L., Simone Di Cola: (Reference) Architecture = Components + Composition $(+$ Variation Points). pp. 1-4 (2015)

12. Paschke, A.P.V.: A reference architecture for event processing. In Proceedings of the Third ACM International Conference on Distributed Event-Based Systems, p. 25 (2009)

13. Len Bass, P.C., Rick, K.: Software Architecture in Practice. Addison Wesley. (2013) 